\title{
Pitch angle diffusion of electrons at the boundary of the lunar wake
}

\author{
Tomoko Nakagawa ${ }^{1}$ and Masahide Iizima ${ }^{2}$ \\ ${ }^{1}$ Tohoku Institute of Technology, 35-1 Yagiyama Kasumi-cho, Taihaku-ku, Sendai, Miyagi 982-8577, Japan \\ ${ }^{2}$ Tohoku University, Aramaki Aoba, Aoba-ku, Sendai, Miyagi 980-8578, Japan
}

(Received December 15, 2004; Revised July 6, 2005; Accepted July 6, 2005)

\begin{abstract}
Velocity distribution of the solar wind electrons that penetrate through the lunar wake boundary is investigated by calculating orbits of the electrons injected into model structures of layers of electric fields. Only the electrons with sufficient energy to overcome the potential difference penetrate through the wake boundary. The electrons injected along the magnetic field lines which intersect the model structure undergo pitch angle scattering due to electric field component perpendicular to the magnetic field. After the passage through the electric field, the electrons have significant perpendicular component of velocity as well as the parallel component larger than a lower limit, which is dependent on the electric potential of the wake boundary. The velocity distribution can account for the cyclotron resonance with sunward-propagating whistler mode waves that were detected by GEOTAIL at 27 lunar radii upstream of the moon on October 25, 1994.
\end{abstract}

Key words: Lunar wake, pitch angle diffusion, electric field, wake potential structure, electron distribution function.

\section{Introduction}

The lunar wake is a plasma cavity in the solar wind left on the anti-solar side of the moon as the solar wind particles are absorbed by the body of the moon (Schubert and Lichtenstein, 1974). The magnetic and plasma fluctuations characteristic of the lunar wake were extensively examined by Explorer 35 (Colburn et al., 1967; Ness et al., 1968). The knowledge on the lunar wake has been refined by WIND spacecraft which traversed the wake at $\sim 6.8$ lunar radii downstream of the moon (Owen et al., 1996). According to the observation by WIND, the plasma density was reduced from ambient solar wind value of about $5-10\left[\mathrm{~cm}^{-3}\right]$ down to $0.5\left[\mathrm{~cm}^{-3}\right.$ ] (Ogilvie et al., 1996; Bosqued et al., 1996). Lunar Prospector also entered into the plasma void repeatedly, and observed depression of the flux of 40-eV electrons by nearly three orders of magnitude (Lin et al., 1998).

At the boundary of the wake, solar wind ions and electrons are thought to rush into the void region at different speeds, producing ambipolar electric field structure. Figure 1 shows a schematic illustration of the structure of the electric potential at the wake boundary. The velocity difference between ions and electrons causes negative excess of charge in the inner region of the wake boundary and positive excess of charge in the outer region of the boundary. These charges produce layers of electric field. When viewed from electrons coming from outside, the potential structure seems as outside traps and inside barrier walls.

Although no direct measurement of such ambipolar electric field has been made, the presence of the ambipolar electric field is suggested by numerical experiments (Farrell et

Copyright (C) The Society of Geomagnetism and Earth, Planetary and Space Sciences (SGEPSS); The Seismological Society of Japan; The Volcanological Society of Japan; The Geodetic Society of Japan; The Japanese Society for Planetary Sciences; TERRAPUB. al., 1998; Birch and Chapman, 2002). On the basis of a simple one-dimensional (1-D) kinetic simulation, Farrell et al. (1998) presented the ambipolar electric field on the flanks of the wake within 5 lunar radii of the moon. In $2 \frac{1}{2} \mathrm{D}$ simulations in the solar wind rest frame, Birch and Chapman (2002) showed the ambipolar electric field pointing radially inward. Similar structure is observed in figure 1 of Guio and Pécseli (2004), although their simulation is not for supersonic flow.

Particle measurements from WIND and NOZOMI also suggest the presence of the ambipolar electric field. In the upstream region of the wake, WIND and NOZOMI detected enhancements of backstreaming electrons with energy of about $500[\mathrm{eV}]$ that are supposed to be reflected at the lunar wake (Farrell et al., 1996; Futaana et al. 2001). During the passage through the wake, WIND detected fieldaligned, cold ion beams refilling the lunar cavity, which were accelerated by an electric field of the order of $2 \times 10^{-4}$ [V/m] (Ogilvie et al., 1996).

Associated with the potential structure at the wake boundary, ULF activities were detected by Explorer 35 (Ness and Shatten, 1969), WIND (Farrell et al., 1996) and GEOTAIL (Nakagawa et al., 2003) on field lines connected to the wake penumbra. From the variation of the observed frequency of the ULF wave that was Doppler-shifted by variable solar wind speed, Farrell et al. (1996) obtained the angular frequency $\omega \simeq-12\left[\mathrm{rad} \mathrm{s}^{-1}\right]$ and the wave number $k \simeq 6 \times 10^{-5}\left[\mathrm{~m}^{-1}\right]$ in the solar wind frame of reference. They concluded that it was a whistler mode wave excited through the cyclotron resonance with reflected electrons. The energy of the resonant electrons was calculated to be 500-1500 [eV]. WIND detected simultaneous enhancement of the backstreaming electrons of the energy of $482[\mathrm{eV}]$. 

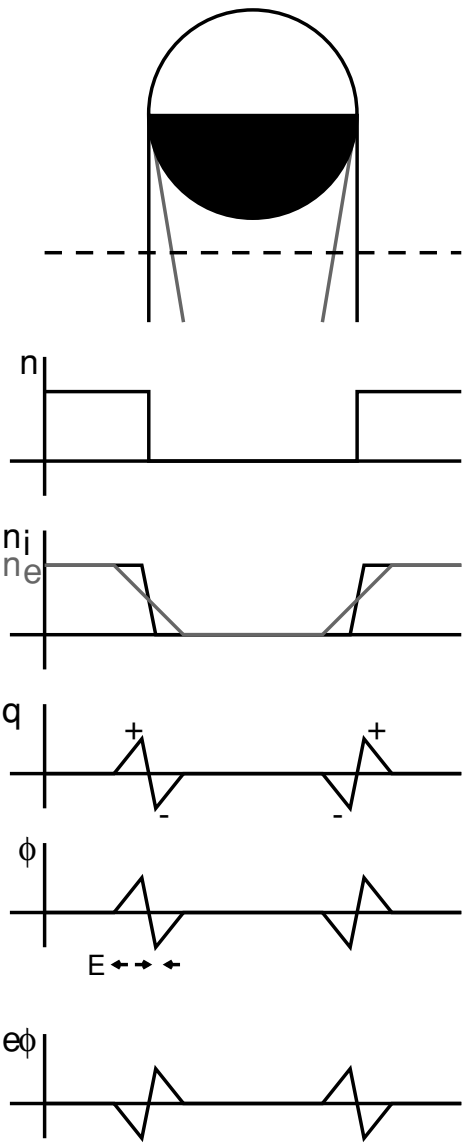

Fig. 1. A schematic illustration of the wake structure. The density profile $n$ along a pass (dashed line) through the wake has a plasma void in the umbra. Different entry speeds of ions and electrons into the void will cause local inequality of ion density $n_{i}$ and electron density $n_{e}$ at the wake boundary, resulting in local inequality of electric charge $q$, which produces electric potential $\phi$ structure. Viewed from electrons coming from outside, the potential structure looks like a combination of an outer trap and an inner wall.

GEOTAIL detected similar but left-handed, circularly polarized ULF waves with frequency of $0.3-1.1[\mathrm{~Hz}]$ at 27 lunar radii upstream of the moon when it was magnetically connected with the lunar wake (Nakagawa et al., 2003). The ULF wave was propagating upstream in the direction nearly parallel to the background magnetic field which intersected the model lunar wake at an angle of 20 degrees. The spectrum of the wave activity had clear, constant upper cutoff at $1.1[\mathrm{~Hz}]$ and variable lower boundary whose frequency was within the range between 0.3 and $1.1[\mathrm{~Hz}]$. The upper cutoff frequency corresponds to the lower limit of the group velocity that can overcome the solar wind velocity to propagate upstream, while the lower boundary seems to indicate the lowest energy of the resonant particles.

The frequency and polarization of the ULF wave are explained by polarity reversal of right-handed, sunwardpropagating electron whistler waves, which were excited through the interaction with electron beams flowing in antisunward direction downstream of the lunar wake (Nakagawa et al., 2003). The angular frequency $\omega$ in the solar wind frame of reference was calculated to be $0.82 \times$ $10^{-2} \Omega_{e}<\omega<2.3 \times 10^{-2} \Omega_{e}$.

The anti-sunward flow of electron beam was explained

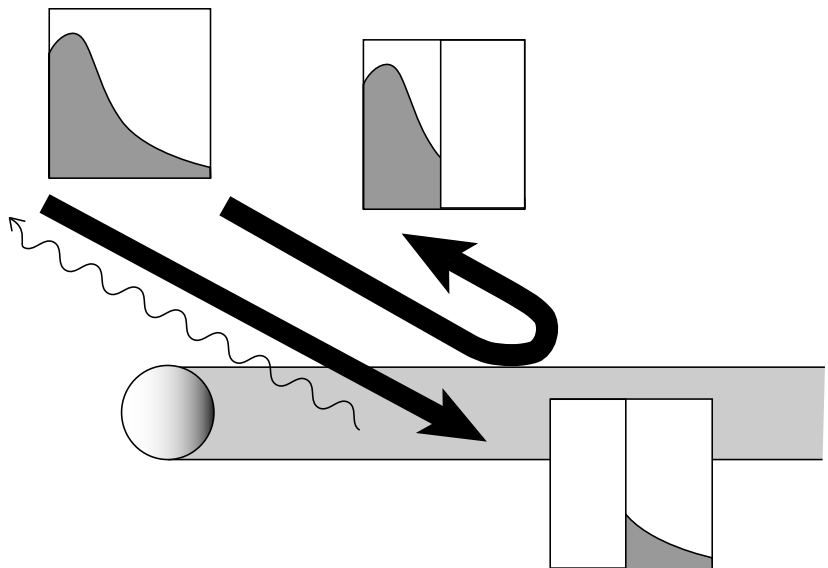

Fig. 2. A schematic illustration of velocity distributions of incoming, reflected, and penetrating electrons. Essentially identical with figure 9 of Nakagawa et al. (2003).

by filtering effect of the potential drop at the boundary of the lunar wake. Figure 2 illustrates the idea. Low-energy components of electrons are reflected back by the potential drop, and the high-energy components, which can overtake the electric potential barrier, can penetrate through the wake. The velocity distribution of downstream electrons would be modified to have some bump or shoulder in energy range, which is likely to excite whistler mode wave through cyclotron resonance. The lowest energy of the resonant electrons was estimated to be 0.96-2.5 [keV] from the lower boundary of the detected frequency (Nakagawa et al., 2003).

In the resonant interactions between waves and electrons, energy can be exchanged between the waves and the particles. The direction of energy transport depends on the initial pitch angle of the electrons. Cyclotron resonance excites whistler mode wave when the electron beam has significant perpendicular component $v_{\perp}$ in velocity space. In the frame of reference of the wave, the energy of resonant electrons is constant, and the resonant electrons move in the $v_{\|}-v_{\perp}$ space along the orbit

$$
\left(v_{\|}-v_{p h}\right)^{2}+v_{\perp}^{2}=\text { const. }
$$

as represented by the circular orbit centered at $\left(v_{p h}, 0\right)$ in velocity space in Fig. 3 . Here $\left(v_{p h}, 0\right)$ is the phase velocity of the wave, and the value of $v_{p h}$ is negative in Fig. 3. If a resonant electron starts with no perpendicular component $v_{\perp}$ and moves along the orbit in Fig. 3(a), its energy increases when viewed from the rest frame. Such electrons extract wave energy, and the wave damps. On the other hand, if the electrons start with significant $v_{\perp}$ (Fig. 3(b)), their energy decreases to excite the wave. In order to excite whistler mode wave, it is required for electrons to have significant velocity component $v_{\perp}$ perpendicular to the magnetic field, as illustrated in Fig. 3(b).

The present paper is going to show that the electrons which penetrate through the lunar wake boundary obtain significant perpendicular component $v_{\perp}$ after the passage through the wake boundary. It will be also shown that the velocity distribution of the penetrating electrons exhibits 
(a)

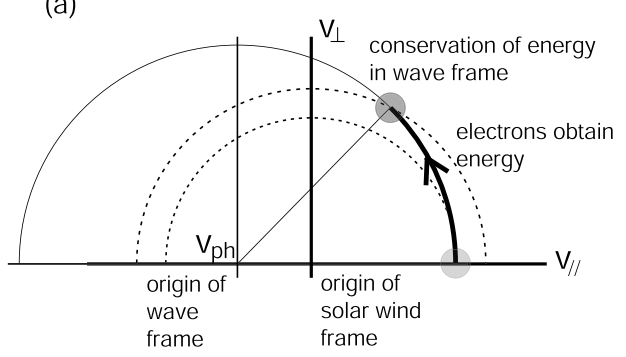

(b)

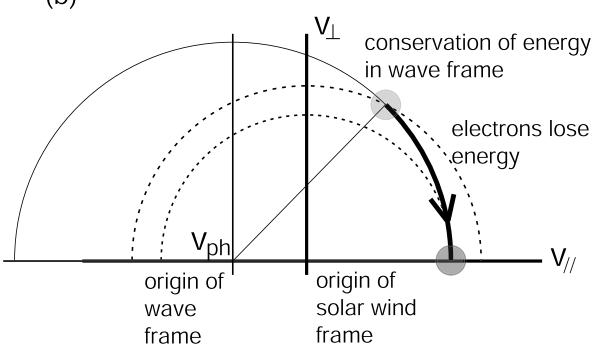

Fig. 3. Resonant conditions in which (a) wave damps or (b) wave is amplified.
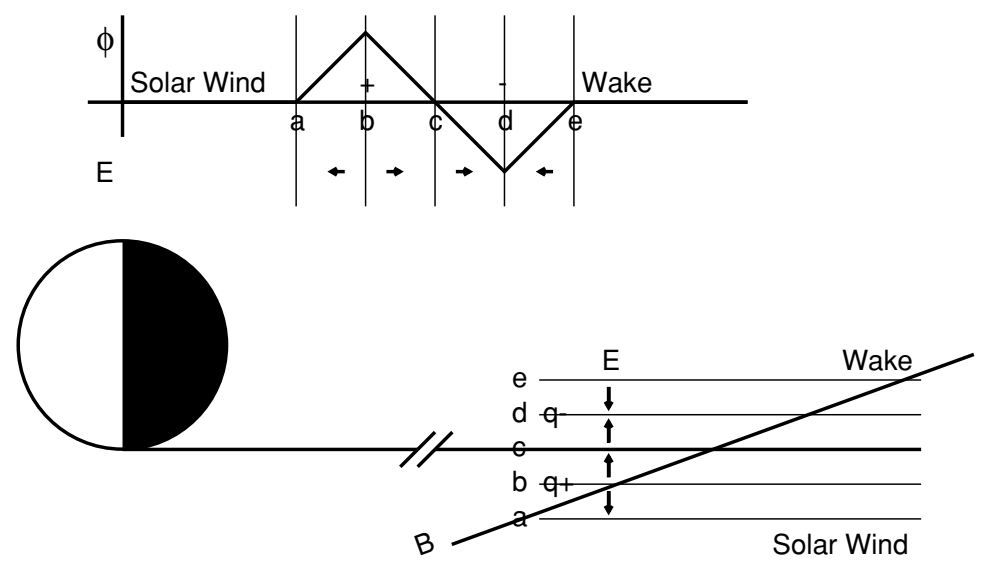

Fig. 4. A simplified model of electric structure at the boundary of the lunar wake. Top: Electric potential $\phi$ and direction of electric field (arrows). Bottom: Layers of electric field at the boundary of the wake.

lower limit in $v_{\|}$, as expected from GEOTAIL observation of the ULF waves associated with the lunar wake.

\section{Test Particle Simulation}

We follow the motion of an electron as a test particle injected into a model structure of electric field by solving the equation of motion

$$
m \frac{d \mathbf{v}}{d t}=q(\mathbf{E}+\mathbf{v} \times \mathbf{B})
$$

together with

$$
\frac{d \mathbf{r}}{d t}=\mathbf{v},
$$

where $m, q, \mathbf{v}, \mathbf{r}$ are the mass, charge, velocity and position of the electron, respectively, $\mathbf{B}$ is the background magnetic field, and $\mathbf{E}$ is the electric field at the wake boundary.

The actual lunar wake is likely to have a cylindrical configuration, but we can safely assume a two-dimensional structure of the electric field if the field is confined in very thin regions with respect to the diameter of the moon. Such confined electric field is observed in Plate 2 of Farrell et al. (1996), and recognized in the potential structures presented in Figure 3 of Birch and Chapman (2001) or in Figure 1 of Guio and Pécseli (2004), in which the electric potential varies in short distance relative to the diameter of the moon. Thus we assume two-dimensional electric field structure in this study.

The electric field is assumed to have layered structure as illustrated in Fig. 4, which corresponds to an expansion of
Fig. 1. Because of the charge inequality, negative excess of charge appears at inner region around 'd' in Fig. 4, and positive charge at outer region around ' $b$ ', producing electric fields directed away from ' $b$ ' toward ' $d$ '. The electric field layers are assumed to be parallel to the wake boundary and the electric field $\mathbf{E}$ is perpendicular to the surface of the layers. In this model, the electric field is confined in between ' $a$ ' and ' $e$ ', and no electric field is assumed outside the layers. The magnetic field is set to intersect the layers at an angle of $20^{\circ}$, which reproduces the configuration of interplanetary magnetic field at the time of GEOTAIL observation of ULF waves (Nakagawa et al., 2003).

We take Cartesian coordinates with its $x$-axis in the direction of the magnetic field $\mathbf{B}=\left(B_{0}, 0,0\right)$, and select $z$ axis so that the $y$-component of electric field vanishes as $\mathbf{E}=\left(E_{x}, 0, E_{z}\right)$. Here $E_{x}$ corresponds to the electric field component $E_{\|}$parallel to the magnetic field, and $E_{z}$ corresponds to the perpendicular component $E_{\perp}$. The electric field vector is assumed to be

$$
\mathbf{E}= \pm E_{0} \mathbf{e}_{E},
$$

where $\mathbf{e}_{E}=\left(\sin 20^{\circ}, 0, \cos 20^{\circ}\right)$ is the unit vector of electric field direction, which is perpendicular to the surface of the wake boundary. The positive/negative sign depends on which layer the electrons is in: it is negative in the layer between ' $a$ ' and ' $b$ ', or between ' $d$ ' and 'e', while it is positive in the layer between ' $b$ ' and ' $d$ '. The intensity of the electric field $E_{0}$ is assumed to be constant just because of the simplicity. It is likely that there is spatial variation in magnitude 
of the electric field in actual lunar wake boundary.

First, we concentrate on the inner two layers between ' $c$ ' and ' $\mathrm{e}$ ' of electric field produced by negative charge, as it seems to play an essential role in filtering electrons according as their energy. Figure 5(a) illustrates the setting of the electric field structure. It is a barrier for electrons injected from the left-hand side of Fig. 5(a). After examining electron behavior in double layered electric field, we proceed to the model structure with three layers as illustrated in Fig. 5(b) to see the role of outer layers between ' $a$ ' and 'c'.

Prior to calculation, Equations (2) and (3) are normalized by the speed $u=E_{0} / B_{0}$, the time $\Omega_{e}^{-1}$, and the length $L=u \Omega_{e}^{-1}$, where $\Omega_{e} \equiv|q \mathbf{B}| / m$ is the electron cyclotron frequency.

\section{Double Layered Model of Electric Field}

First, we observe variation of velocity of electrons in a double-layered structure of electric field

$$
\mathbf{E}= \begin{cases}+E_{0} \mathbf{e}_{E} & \left(0<\mathbf{r} \cdot \mathbf{e}_{E}<d\right) \\ -E_{0} \mathbf{e}_{E} & \left(d<\mathbf{r} \cdot \mathbf{e}_{E}<2 d\right)\end{cases}
$$

as illustrated in Fig. 5(a). Thickness of each layer $d$ is set to be $1.5 L$ for this example.

Figure 6 shows an example orbit of an electron injected into the double-layered structure along the background magnetic field B. Left two panels of Fig. 6 show the trajectory in velocity space, and the right two panels are for configuration space. As the initial velocity $\mathbf{v}_{0}=(2.5 u, 0,0)$ is parallel to $\mathbf{B}$, the test particle is on the origin in $v_{y}-v_{z}$ space at the beginning. Here red color indicates that the electron is in the outer layer of inward electric field, while blue color is for the inner layer of outward electric field. Green color is for regions of no electric field.

As the test particle enters into the layer of inward electric field (red), it starts drift motion with drift speed $u_{D}=$ $E_{\perp} / B_{0}=u \cos 20^{\circ}$, getting perpendicular component $v_{\perp}$ of velocity. Due to the perpendicular component $v_{\perp}$, the electron begins to gyrate around the magnetic field, and

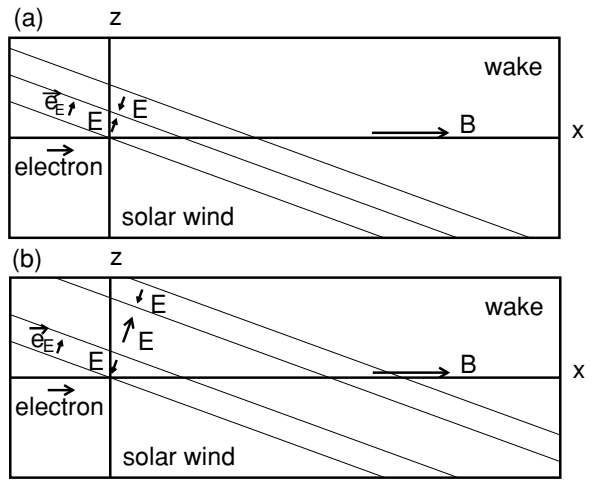

Fig. 5. (a) The setting of the double-layered model structure of the inward electric field in the outer layer and the outward electric field in the inner layer, which corresponds to layers between 'c' and 'e' in Fig. 4. (b) The triple-layered structure of electric field which corresponds to layers between ' $a$ ' and 'e' in Fig. 4. The intensity of the electric field $E_{0}$ is assumed to be constant just because of the simplicity. Electrons are injected from the left-hand side. traces a circular orbit centered at $\left(u_{D}, 0\right)$ with radius $u_{D}$ in $v_{y}-v_{z}$ space. The perpendicular speed $v_{\perp}$ may become as much as $2 u_{D}$ after $(2 n+1) \pi$ gyration $(n=0,1,2, \ldots)$. The magnitude of $v_{\perp}$ gained by the electron in this layer depends on the phase of gyration at the moment of exit from this layer.

At the same time, the parallel component $E_{\|}$of the electric field acts on the electron to decelerate it in $x$ direction. The second left panel of Fig. 6 shows decrease of $v_{\|}$and increase of $v_{\perp}$ in the layer of the inward electric field (red). From the conservation of energy

$$
\frac{1}{2} m v_{0}^{2}=q \Delta \phi+\frac{1}{2} m v^{2},
$$

the speed $v$ of the electron becomes minimum

$$
v=\sqrt{v_{0}^{2}-v_{c}^{2}}
$$

at the moment when it reached the end of the first layer with potential drop $\Delta \phi$. Here

$$
v_{c}=\sqrt{\frac{2 q \Delta \phi}{m}},
$$

is the critical speed to climb up the potential difference $\Delta \phi$. For the present case, $v_{0}=2.5 u$ and $\Delta \phi=E_{0} d=1.5 L E_{0}$, the critical speed $v_{c}$ is $\sqrt{3} u$, and the minimum speed $v$ is about $1.8 u$.

The electron that has climbed up the potential difference then enters the second layer of outward-directed electric field (blue), where the drift speed becomes negative. The electron begins to gyrate around $\left(-u_{D}, 0\right)$ in $v_{y}-v_{z}$ space, and the perpendicular speed $v_{\perp}$ increases.

At the same time, the electron is accelerated in $x$ direction because of the presence of the parallel component $E_{\|}$ of electric field. The electron regains the kinetic energy that was converted into potential energy.

After gyrating about $\pi / 4$ in the second layer, the electron gets out of the electric field with significant perpendicular speed $v_{\perp}$. After a shallow entry into the second layer, it leaves the boundary and enters the central void of the wake. The final velocity of the electron is $v_{\perp}=2.06 u$ and $v_{\|}=1.42 u$, that is, $v=2.5$ with the pitch angle of $55^{\circ}$. The pitch angle is largely diffused, while the kinetic energy is conserved.

Similar calculations are made for a number of electrons with various initial speeds and pitch angles. Figure 7 shows initial and final velocity distributions. The electrons are injected into the double-layered electric field nearly along the magnetic field with various initial velocities

$$
\mathbf{v}_{0}=v_{0}\left(\begin{array}{l}
\cos \theta_{0} \\
\sin \theta_{0} \cos \phi_{0} \\
\sin \theta_{0} \sin \phi_{0}
\end{array}\right),
$$

where $v_{0}$ is the initial speed ranging from $0.02 u$ to $5 u$, and the initial pitch angle $\theta_{0}$ ranges from $0^{\circ}$ to $10^{\circ}$ as measured from the direction of the magnetic field. The initial phase of gyration, $\phi_{0}$, ranges from $0^{\circ}$ to $180^{\circ}$. Each of the test particles represents electrons in each solid angle $0.04 u \times$ $1^{\circ} \times 1^{\circ}$. After calculation of their orbits, the number of test 

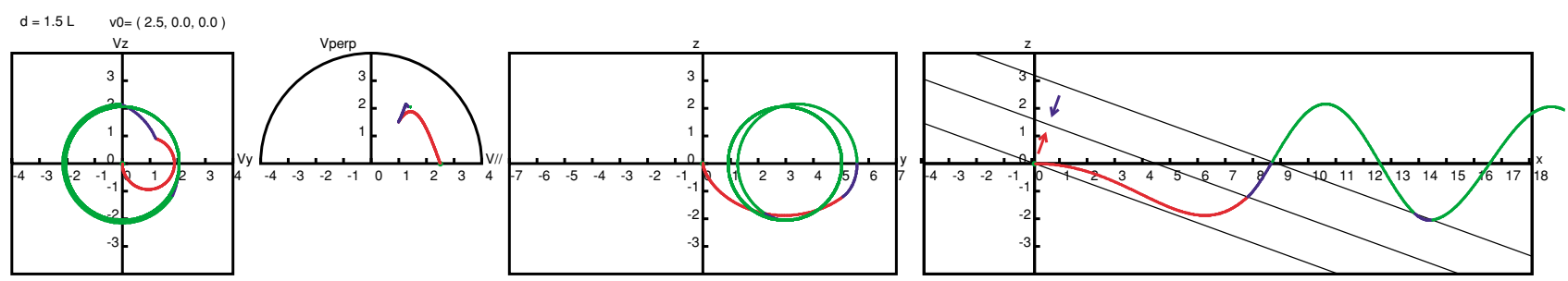

Fig. 6. An example of the trajectory of an electron with initial velocity $\mathbf{v}_{0}=(2.5 u, 0,0)$, injected into a double layered model with $d=1.5 L$ along the magnetic field line. From left to right: the trajectory in velocity space $v_{y}-v_{z}$ perpendicular to the magnetic field, the trajectory in $v_{\|}-v_{\perp}$ space, the orbit in configuration space $y-z$ perpendicular to the magnetic field, and that in configuration space $x-z$ where $x$ axis is parallel to the magnetic field line. Red color indicates that the test particle is in the first layer of electric field, while blue color in the second layer of the reversed electric field. Green color indicates that the particle is out of the electric fields.
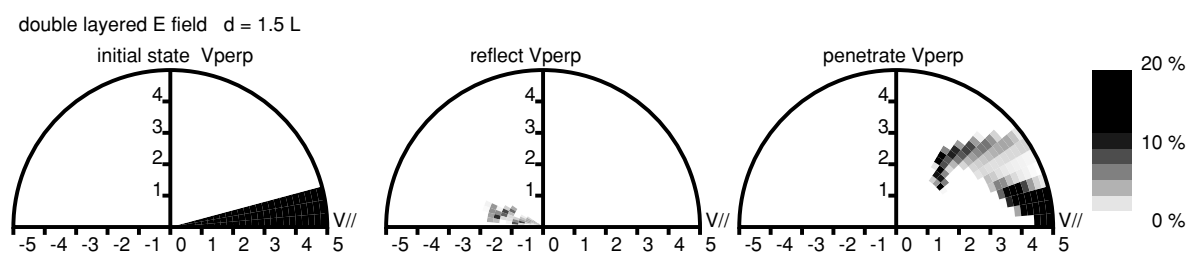

Fig. 7. Velocity distributions of the incident electrons (left panel), of the electrons reflected by the electric field (center panel), and of the electrons which passed through the double layered model electric field (right panel). The electric field is represented by Eq. (5). The initial speed is in the range $0.02 u-5 u$ and the pitch angle is between $0^{\circ}$ and $10^{\circ}$ from the direction of the magnetic field.

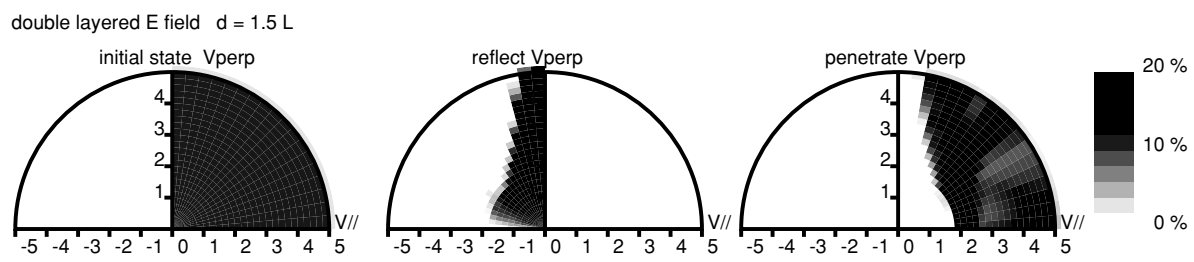

Fig. 8. Same as Fig. 7 but the initial pitch angles are distributed wider, between $0^{\circ}$ and $90^{\circ}$ from the magnetic field.

particles multiplied by each solid angle is counted for each bins of $0.2 u \times 1^{\circ} \times 1^{\circ}$.

The final velocity distribution is presented separately in two panels, one is for electrons reflected back by the potential barrier (middle panel of Fig. 7), and the other is for those which penetrate through the wake boundary (right panel of Fig. 7). During the passage through the second layer, the electrons that penetrate through the wake regain the kinetic energy that was converted into potential energy, and their speed become larger than the critical speed $\left(v_{c}=\sqrt{3} u\right.$ for this case) in the downstream of the boundary. As they are accelerated in $x$ direction in the second layer due to the presence of $E_{\|}$, they obtain non-zero $v_{\|}$in the downstream region. We observe a lower limit of $v_{\|}$at around $1.2 u$ in the right panel of Fig. 7. At the same time, the pitch angle is largely diffused from the initial state. The penetrating electrons gain perpendicular component $v_{\perp}$ as much as $2.5 u$.

The lower limit of $v_{\|}$is clearer in Fig. 8, which shows the results for electrons with wider range of initial pitch angle. During the passage through the second layer of outward electric field, the penetrating electrons are accelerated in $x$ direction, and obtain a finite $v_{\|}$. Slowest particle stays for longest time in the layer being accelerated, thus the electrons have significant $v_{\|}$downstream of the wake boundary.

\section{Triple Layered Model of Electric Field Struc- ture}

Next we proceed to the model of triple-layered structure

$$
\mathbf{E}=\left\{\begin{array}{cc}
+E_{0} \mathbf{e}_{E} & \left(0<\mathbf{r} \cdot \mathbf{e}_{E}<d\right) \\
-E_{0} \mathbf{e}_{E} & \left(d<\mathbf{r} \cdot \mathbf{e}_{E}<3 d\right) \\
+E_{0} \mathbf{e}_{E} & \left(3 d<\mathbf{r} \cdot \mathbf{e}_{E}<4 d\right)
\end{array}\right.
$$

as illustrated in Fig. 5(b). Again the thickness $d$ is set to be $1.5 \mathrm{~L}$. Figure 9 compares trajectories of an electron in the triple-layered structure and in the double-layered structure. The initial speed $v_{0}$ is $3.5 u$ and the initial pitch angle is $10^{\circ}$. In the double-layered model (upper panels), the perpendicular component $v_{\perp}$ becomes $1.5 u$ after the passage through the layered electric fields, while in the triplelayered model (lower panels), the electron gains $v_{\perp}$ as much as 2.6u. Entering into the first layer of outward electric field (blue) of the triple-layered model, the electron begins to rotate around $\left(-u_{D}, 0\right)$ in $v_{y}-v_{z}$ space. It gives the electron a chance to obtain larger speed with respect to the point $\left(u_{D}, 0\right)$, the center of gyration of the next layer. In the second layer of inward electric field (red), it rotates around $\left(u_{D}, 0\right)$ with the large relative speed gained in the first layer. Finally it enters into the last layer (blue) with $v_{\perp} \sim 2.3 u$ and gyrate around $\left(-u_{D}, 0\right)$, then exit with large $v_{\perp} \sim 2.6 u$. 


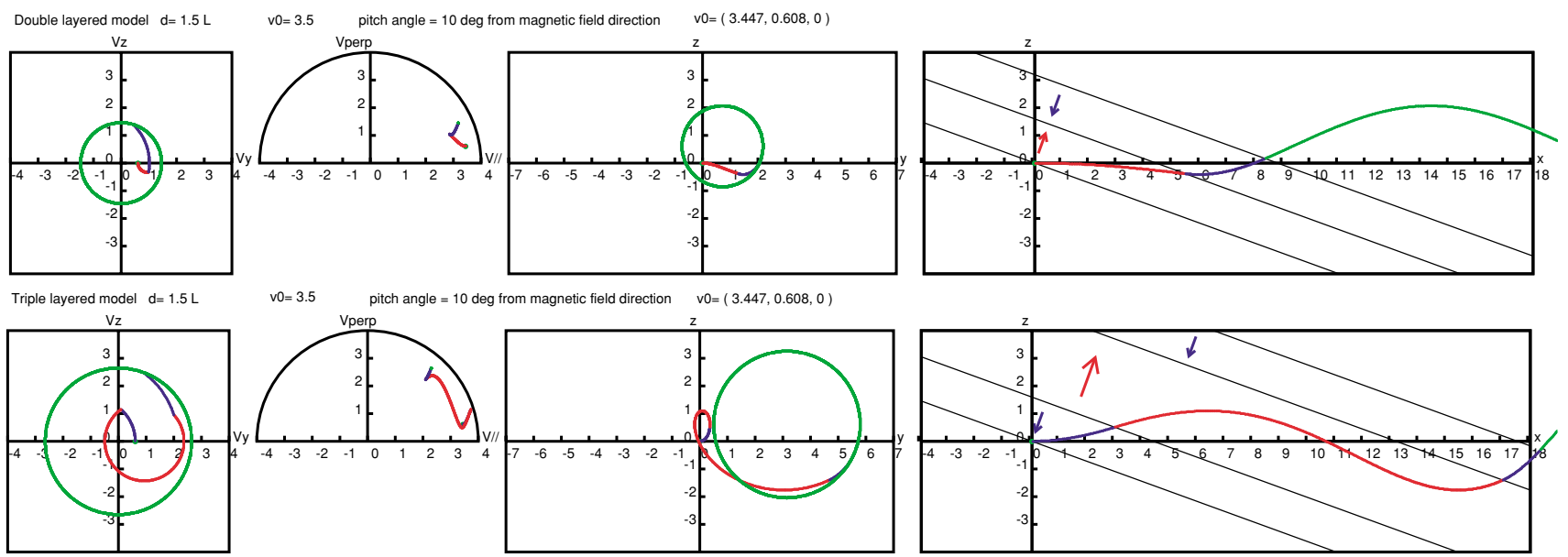

Fig. 9. Comparison of electron trajectories in double-layered and triple-layered structure of electric field, with the same initial speed $v_{0}=3.5 u$ and pitch angle $10^{\circ}$. The format is the same as that of Fig. 6 .

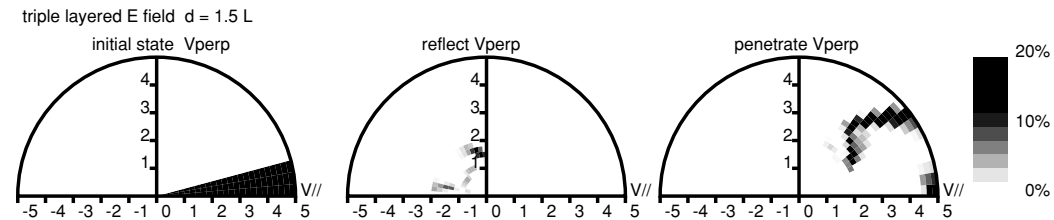

Fig. 10. Velocity distributions of the incident electrons (left panel), of the electrons reflected by the electric field (center panel), and of the electrons which passed through the triple layered model electric field (right panel). The electric field is represented by Equation (10). The initial speed is in the range $0.02 u-5 u$ and the pitch angle is between $0^{\circ}$ and $10^{\circ}$ from the direction of the magnetic field.

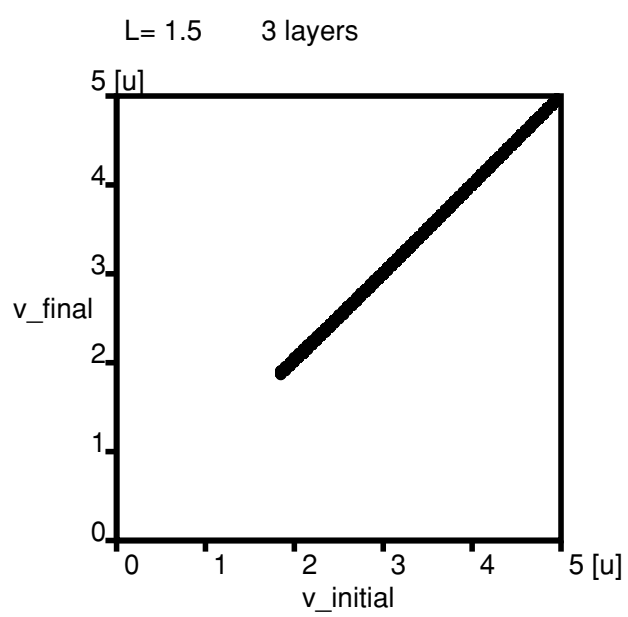

Fig. 11. The final speeds versus the initial speeds of test-particle electrons after the passage through the triple-layered structure of the electric field. This plot is produced by calculating velocity of particles for various speed $0.05 u<v_{0}<5 u\left(\Delta v_{0}=0.05 u\right)$, pitch angle $0<\theta<10^{\circ}$ $\left(\Delta \theta=1^{\circ}\right)$ and phase angle $0<\phi<180^{\circ}\left(\Delta \phi=2^{\circ}\right)$.

Figure 10 shows final states of electrons injected into triple-layered electric fields with various initial speeds ranging from $0.02 u$ to $5 u$ and pitch angle between $0^{\circ}$ and $10^{\circ}$. The pitch angle becomes larger in the triple-layered structure than in the double-layered electric fields in Fig. 7. It is also recognized that the lower limit of $v_{\|}$is higher in the triple layered model than in the double layered model. The outer trapping electric field contributes to the pitch an- gle diffusion by giving another chance of transition between layers of different drift velocities.

The initial kinetic energy of test-particle electron is conserved after the passage through the layered structure of the electric field. Figure 11 shows the final speeds of the electrons that passed though the triple-layered electric field, as a function of their initial speeds ranging from $0.05 u$ to $5 u$ with initial pitch angles of $0^{\circ}-10^{\circ}$. The conservation of the kinetic energy is recognized. The blank area of lowenergy range is for the particles reflected back, not penetrating through the layers.

\section{Thickness of the Layers of Electric Fields}

Figure 12 shows the velocity distribution of the electrons after the passage through the triple-layered models of various thickness $d=0.3 L, 0.6 L, L, 2 L$, or $3 L$. Test particles are injected nearly parallel to the magnetic field, with pitch angle of $0^{\circ}-10^{\circ}$.

In the thin layered models with $d=0.3 L$ or $0.6 L$, few electrons obtain large $v_{\perp}$. The electrons easily get out of the layer before they gyrate significant phase angle to obtain large $v_{\perp}$. The lower limit of $v_{\|}$is not very clear, either.

In the electric field model with each layer as thick as $d \sim L$, the electrons spend longer time in each layer than in the thin models, and gyrate larger angle in cyclotron motion. Some of the slow-starting electrons gyrate as much phase angle as $\pi$ by the time they exit the layer, and obtain maximum perpendicular speed $v_{\perp}$ in the layer.

In thicker models, e.g., $d>2 L$, the slowest electrons 
can gyrate by the phase angle of $2 \pi$, getting back to the origin in $v_{y}-v_{z}$ space. According as the phase angle of the gyration becomes $3 \pi, 4 \pi, 5 \pi, \ldots$, the perpendicular speed of electrons becomes maximum, 0 , maximum, ..., alternately. The dependence of $v_{\perp}$ on $v_{\|}$is recognized in the bottom 2 panels of Fig. 12. The electrons with large $v_{\perp}$ is clustered in some bands of $v_{\|}$. If such electrons excite waves which are to be observed by GEOTAIL, it is likely that the whistler mode wave has some band structure in its spectrum. As far as the waves observed by GEOTAIL is concerned, no such signature has been detected.

It is also recognized in Fig. 12 that the lower limit of $v_{\|}$increases with the thickness of the electric field structure. The relationship between the lower limit of $v_{\|}$and the thickness is summarized in Table 1, which shows that the lower limit of $v_{\|}$is of the order of the critical speed $v_{c}$.

\section{Discussion}

\subsection{Summary of experiment}

The results of the test-particle simulation are summarized as follows:
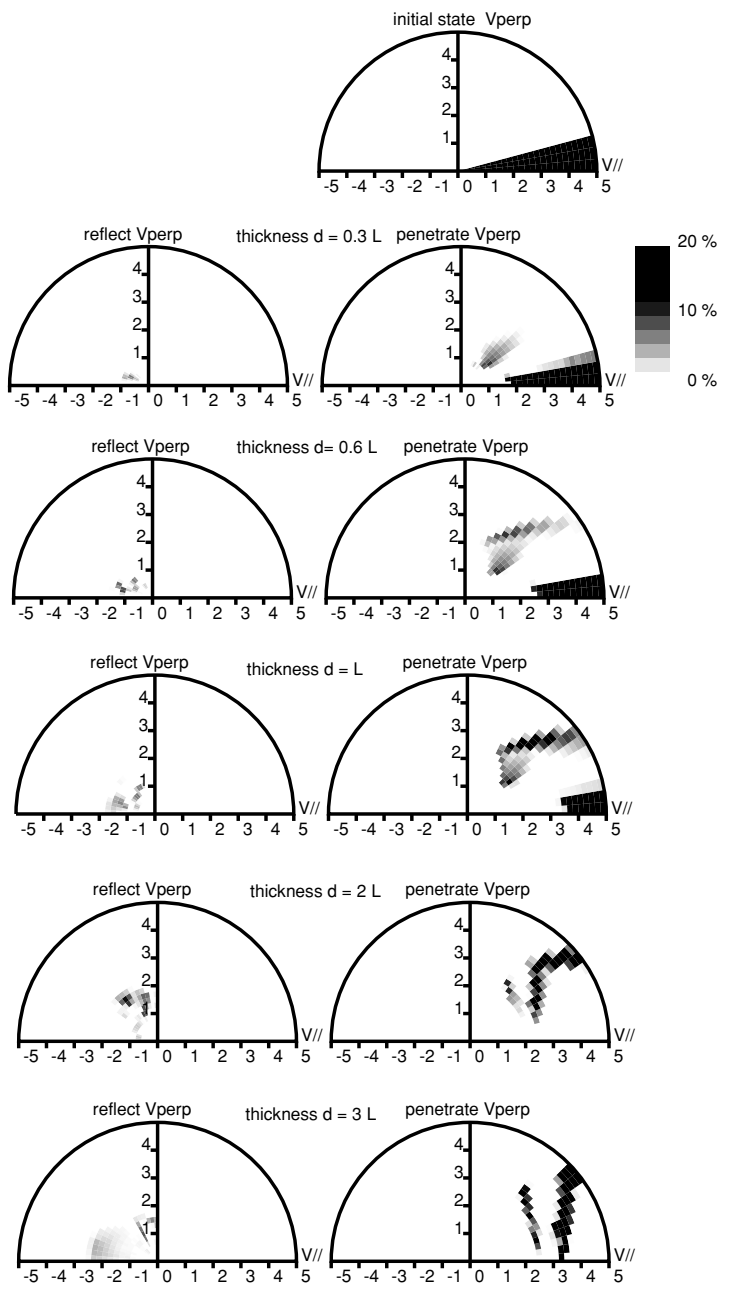

Fig. 12. Effect of variation of the thickness of the layer of electric field. Top panel shows the initial states, and the followings show the final states of electrons that are reflected by, or penetrating through triple-layered structures with thickness of $d=0.3 L, 0.6 L, 1.0 L, 2.0 L$, and $3.0 \mathrm{~L}$.
Table 1. Lower limit of $v_{\|}$after the passage through the triple layered electric fields of various thickness.

\begin{tabular}{lcc}
\hline thickness $d$ & lower limt $v_{\|}$ & $v_{c}$ \\
\hline $0.1 L$ & $0.5 u$ & $0.4 u$ \\
$0.3 L$ & $0.7 u$ & $0.8 u$ \\
$0.4 L$ & $0.7 u$ & $0.9 u$ \\
$0.7 L$ & $0.7 u$ & $1.2 u$ \\
$0.8 L$ & $1.0 u$ & $1.3 u$ \\
$L$ & $1.2 u$ & $1.4 u$ \\
$1.5 L$ & $1.6 u$ & $1.7 u$ \\
$2 L$ & $1.3 u$ & $2.0 u$ \\
$3 L$ & $2.0 u$ & $2.4 u$ \\
$4 L$ & $2.5 u$ & $2.8 u$ \\
\hline
\end{tabular}

i) The inward electric field produced by negative excess of charge first decelerates incoming electrons and reflects those with less energy than that of potential energy. The electric field structure at the lunar wake boundary behaves like a filter which allows only high energy electrons to pass through and retard low energy electrons.

ii) In the inner layer, the component $E_{\|}$of the outwarddirected electric field produced by negative excess of charge then accelerates the electrons which penetrate through the wake boundary toward the central void. Thus the electrons regain the kinetic energy which was spent in climbing the potential difference. Thus in the downstream, the parallel component $v_{\|}$of electron velocity becomes larger than a lower limit.

iii) The electric field component $E_{\perp}$ perpendicular to the magnetic field causes $\mathbf{E} \times \mathbf{B}$ drift of electrons in the wake boundary. The electrons obtain significant velocity component $v_{\perp}$ due to the electric-field drift.

iv) Through the transition between layers of electric field with different drift speed, some of the penetrating electrons obtain more perpendicular speed $v_{\perp}$.

v) The electric field produced by positive charge in the outer layer of the lunar wake contribute to larger diffusion of pitch angle of electrons, by giving more chance of transit between layers of different drift speeds.

\subsection{Origin of the penetrating electrons}

The present simulation shows that the initial kinetic energy of electrons are conserved after the passage through the wake boundary. On the other hand, the GEOTAIL observation suggested that the minimum energy of the penetrating electrons was in the range from $0.96[\mathrm{keV}]$ to 2.5 [keV] (Nakagawa et al., 2003). Thus the incident electrons are expected to have as much energy.

The electrons of such energy range are often observed in the solar wind and referred to as the halo component (Feldman et al., 1975; Gosling et al., 1987). When the halo component flowing outward from the sun is narrowly 


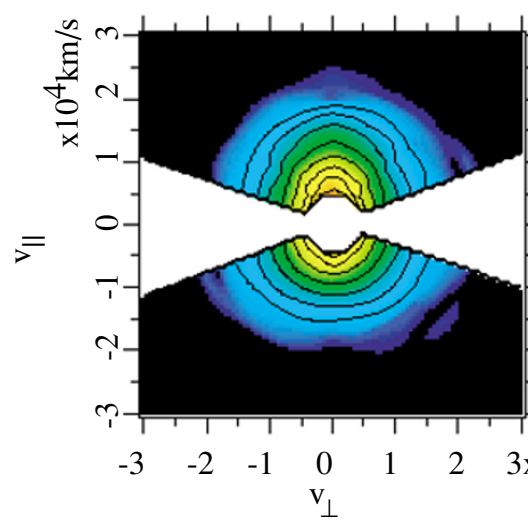

(in direction of electric field $\mathrm{E}$ )

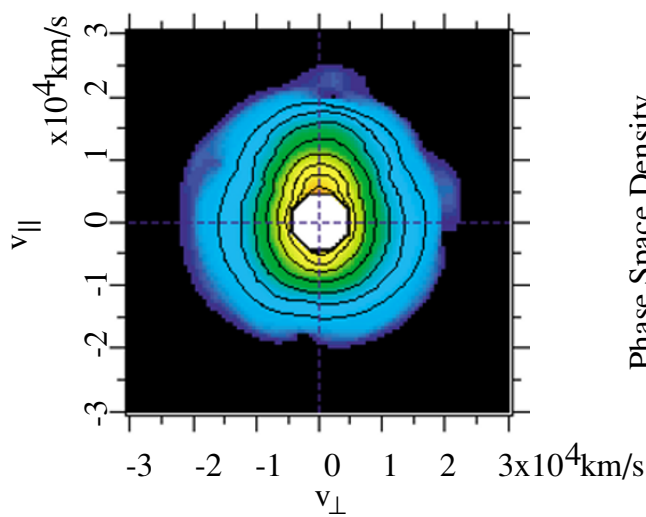

(in direction of convection $\mathrm{ExB}$ )
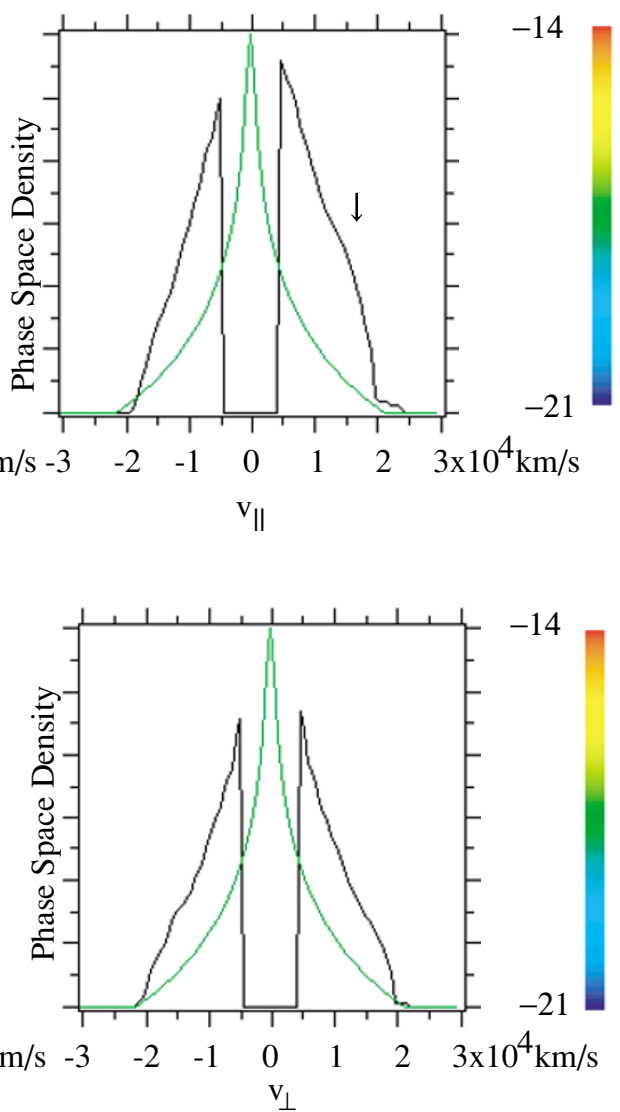

(in direction of convection flow)

Fig. 13. An example of the velocity distribution of the solar wind electrons obtained by GEOTAIL/LEP on October 25, 1994, 16:54:52-16:55:52 UT. Left panels show contours of the distribution function in $v_{\|}-v_{\perp}$ planes, where the upper left panel shows $v_{\perp}$ in the direction of electric field $\mathbf{E}$, while the lower left panel shows $v_{\perp}$ in the direction of the convection velocity $\mathbf{E} \times \mathbf{B}$. Observation of low energy components in the energy regime of core electrons was not carried out in the sweep mode (RAM-A), as indicated by the central blank area in the left panels. Upper right panel shows cut of the distribution function along the $v_{\|}$axis indicated by the vertical dashed line in the lower-left panel, while the lower right panel is the cut at the $v_{\perp}$ axis indicated by the horizontal dashed line in the lower-left panel. Green curves in the right panels show the one-count limits, that is, the least level required for incoming electrons to produce the least bit signal. In the upper right panel, an enhancement of strahl component is observed at around $1.5 \times 10^{4}-2.0 \times 10^{4}[\mathrm{~km} / \mathrm{s}]$ which corresponds to $1.0-1.8[\mathrm{keV}]$, as marked with an arrow. In this panel, positive $v_{\|}$corresponds to electron flow away from the sun, since the magnetic field was directed away from the sun.

distributed along the magnetic field, it is referred to as the strahl component (Pilipp et al., 1987; Phillips et al., 1989; McComas et al., 1989).

During the period of GEOTAIL detection of ULF waves associated with the lunar wake boundary, the spacecraft observed halo electrons with its peaks at around $1[\mathrm{keV}]$ in the solar wind far upstream of the moon (27 lunar radii). Figure 13 is one of the examples of the electron distribution function obtained by GEOTAIL/LEP during the detection of the ULF wave. It shows only halo components because the lowest energy step of the operation mode (RAM-A) of LEP is centered at 60 [eV] (Mukai et al., 1994). The left panels show cross sections of the distribution function in $v_{\|}-v_{\perp}$ planes, and the right two panels show profiles of the distribution function along one of the dashed lines in the lower left panel. In the upper right panel, the strahl component is recognized at around $v_{\|} \sim 1.5 \times 10^{4}-2.0 \times$ $10^{4}[\mathrm{~km} / \mathrm{s}]$, which corresponds to $1.0[\mathrm{keV}]-1.8[\mathrm{keV}]$. As the magnetic field was directed away from the sun at this time, positive $v_{\|}$corresponds to electron flow away from the sun, in agreement with the antisunward flow of strahl electrons along the magnetic field. On the other hand, no such enhancement is seen in the cut along the $v_{\perp}$ axis in the lower right panel. Thus the origin of the penetrating electrons is thought to be the strahl component of solar wind electrons flowing downstream along the magnetic field.

\subsection{An attempt to estimate the thickness of the layer}

The energy of electrons that penetrate through the wake boundary is estimated from the lower cutoff frequency of the ULF waves detected by GEOTAIL (Nakagawa et al., 2003). The lower limit of $v_{\|}$, which is of the order of $v_{c}$, gives a measure of $\Delta \phi$, but it gives no restriction on the thickness $d$ of the boundary layer.

In order to estimate the thickness of the wake boundary structure, we re-examine the velocity distribution in Fig. 12. Excitation of wave through cyclotron resonance requires significant perpendicular speed $v_{\perp}$, while the electrons do not gain much perpendicular speed $v_{\perp}$ in thin models of $d<L$. Thus it seems that the thickness of each layer of the lunar wake boundary is larger than $L$.

As the layer becomes thicker, $d>2 L$, the lower limit of $v_{\|}$becomes higher, but the upper limit of $v_{\perp}$ is restricted around $4 u_{D}$ for the case of the triple layered model. Thus in the thicker model, we cannot expect high $v_{\perp} / v_{\|}$ratio 
which is important for wave excitation through cyclotron resonance. Thus it seems likely that the thickness of the boundary was between the range from $d \sim L$ to $2 L$ for the GEOTAIL observation.

The most effective thickness $d \sim 1-2 L$ for pitch angle diffusion, where $L=u \Omega_{e}^{-1}$, is nearly equal to the Larmor radius $r_{L}=v_{\perp} \Omega_{e}^{-1}$ of the penetrating electrons that was initially injected nearly parallel to the magnetic field, as $v_{\perp}$ is of the order of $u_{D}-3 u_{D}$ (typical value recognized in Fig. 10).

As the length $L=u \Omega_{e}^{-1}=E_{0} B_{0}^{-1} \Omega_{e}^{-1}$ is related with the electric field, and the electric field is written in the form of

$$
E_{0}=\frac{\Delta \phi}{d},
$$

our estimation of the thickness $d=(1 \sim 2) L$ turns to be

$$
d=(1 \sim 2) \frac{\Delta \phi}{B_{0} \Omega_{e} d},
$$

and we obtain

$$
d=\sqrt{(1 \sim 2) \frac{\Delta \phi}{B_{0} \Omega_{e}}} .
$$

According to the GEOTAIL observation, $B_{0}=6[\mathrm{nT}]$, $\Omega_{e}=2 \pi \times 174[\mathrm{~Hz}]$, and $\Delta \phi=0.96-2.5[\mathrm{keV}]$, we obtain the thickness of a single layer about $12-28[\mathrm{~km}]$ and the whole thickness of multi-structure about 48-110 $[\mathrm{km}]$. The magnitude of the electric field is calculated to be around $80[\mathrm{mV} / \mathrm{m}]$. Assuming that the potential structure is a result of velocity difference of ions and core components of electrons, with thermal speeds of $50[\mathrm{~km} / \mathrm{s}](20[\mathrm{eV}])$ and $5000[\mathrm{~km} / \mathrm{s}](80[\mathrm{eV}])$, respectively, it takes only $0.01-0.02$ sec for the electrons to traverse the distance into the void, and during the time the solar wind flows by $500[\mathrm{~km} / \mathrm{s}] \times$ $(0.01-0.02)[\mathrm{sec}]=5-10[\mathrm{~km}]$. Thus, the wake potential structure which disturbed high energy electrons to excite whistler wave detected by GEOTAIL is supposed to be in close vicinity $(\sim 10[\mathrm{~km}])$ of the moon.

\section{Conclusion}

The layered structure of electric field at the boundary of the lunar wake modifies the velocity distribution of incoming electrons to exhibit lower limit of parallel component $v_{\|}$ and to have significant perpendicular component $v_{\perp}$ which is expected to excite waves through cyclotron resonance. The velocity distribution obtained in this study is favorable to the generation of the whistler mode wave observed by GEOTAIL in association with the magnetic connection with the lunar wake (Nakagawa et al., 2003).

The electric field component $E_{\|}$parallel to the magnetic field contributes to reflection of low-energy electrons and modification of the electron distribution function to exhibit lower limit in $v_{\|}$, while the electric field component $E_{\perp}$ perpendicular to the magnetic field contributes to the pitch angle diffusion through the electric field drift. In the absence of the electric field component $E_{\|}$parallel to the magnetic field, there will be no lower limit in the distribution of $v_{\|}$. Even in such a case, the pitch angle diffusion in $E_{\perp}$ makes it possible for electrons with such a ring-shaped velocity distribution function to excite a wave.
In this study, we assumed double- or triple-layered structures of electric field with equal amount of potential drop and potential rise, resulting in equal electric potential between the solar wind and the central part of the wake. At the real wake boundary, it is likely that the magnitude of the electric field as well as the thickness of the electric field layer is different for each layer. For example, if inward electric field dominates outward directed fields, and the electric potential in the central wake is negative, the kinetic energy of the penetrating electrons would be smaller than that of the solar wind, and the low-energy cutoff in $v_{\|}$would be less significant. The pitch angle diffusion is again effective even in such cases.

One might think that the field-aligned electric field can easily be cancelled by cold component of the plasma, but it takes time for the electrons to fulfill the void while the solar wind plasma flows down, thus there is an electric field component perpendicular to the solar wind flow in close vicinity of the moon. When the magnetic field is not parallel to the solar wind flow, as in the case of GEOTAIL observation (Nakagawa et al., 2003), there must be the electric field component $E_{\|}$parallel to the magnetic field. The proximity of the region of the electric field is consistent with the discussion in Section 6.3.

On the other hand, if the magnetic field happens to be parallel to the solar wind flow, entry of suprathermal electrons along the magnetic field is not expected. In such cases, difference of gyroradii of ions and electrons might be essential. Thus the direction of magnetic field is thought to be of great importance for the electric field structure at the wake boundary.

Although Nakagawa et al. (2003) assumed a whistler mode wave excited in the wake, it is not known what wave mode is really excited. The wave detected by GEOTAIL matched the profile of the whistler mode wave for the very parameters of the solar wind observed by GEOTAIL, on the other hand, previous observations in the lunar wake showed that the plasma density was depressed in the wake. Thus the dispersion relation of the whistler mode in the wake must be different from that in the solar wind. Whatever mode in the wake is, a wave, once launched upstream into the solar wind, propagates as the whistler mode wave only if the wave frequency at the boundary of the wake is in the range allowed for the whistler mode wave in the solar wind frame. For the case of GEOTAIL observation, the range of angular frequency is $0.82 \times 10^{-2} \Omega_{e}<\omega<2.3 \times 10^{-2} \Omega_{e}$. Mode conversion may occur at the boundary. Thus knowing the mode of the wave excited in the wake is subject to the future work.

Acknowledgments. The authors are grateful to T. Mukai and GEOTAIL/LEP team for the electron distribution function presented in Fig. 13. Thanks are also due to T. Hada and S. Machida for valuable comments and discussions.

\section{References}

Birch, P. C. and S. C. Chapman, Correction to "Particle-in-cell simulations of the lunar wake with high phase resolution", Geophys. Res. Lett., 28, 2669, 2001.

Birch, P. C. and S. C. Chapman, Two dimensional particle-in-cell simulations of the lunar wake, Phys. Plasmas, 9, 1785-1789, 2002.

Bosqued, J. M., N. Lormant, H. Rème, C. d'Uston, R. P. Lin, K. A. 
Anderson, C. W. Carlson, R. E. Ergun, D. Larson, J. McFadden, M. P. McCarthy, G. K. Parks, T. R. Sanderson, and K.-P. Wenzel, Moonsolar wind interaction: First results from the WIND/3DP experiment, Geophys. Res. Lett., 23, 1259-1262, 1996.

Colburn, D. S., R. G. Currie, J. D. Mihalov, and C. P. Sonett, Diamagnetic solar-wind cavity discovered behind moon, Science, 158, 1040-1042, 1967.

Farrell, W. M., R. J. Fitzenreiter, C. J. Owen, J. B. Byrnes, R. P. Lepping, K. W. Ogilvie, and F. Neubauer, Upstream ULF waves and energetic electrons associated with the lunar wake: Detection of precursor activity, Geophys. Res. Lett., 23, 1271-1274, 1996.

Farrell, W. M., M. L. Kaiser, J. T. Steinberg, and S. D. Bale, A simple simulation of a plasma void: Applications to Wind observations of the lunar wake, J. Geophys. Res., 103, 23635-23653, 1998.

Feldman, W. C., J. R. Asbridge, S. J. Bame, M. D. Montgomery, and S. P. Gary, Solar wind electrons, J. Geophys. Res., 80, 4181-4196, 1975.

Futaana, Y., S. Machida, T. Saito, A. Matsuoka, and H. Hayakawa, Counterstreaming electrons in the near vicinity of the moon observed by plasma instruments on board NOZOMI, J. Geophys. Res., 106, 18729_ 18740, 2001

Gosling, J. T., D. N. Baker, S. J. Bame, W. C. Feldman, R. D. Zwickl, and E. J. Smith, Bidirectional solar wind electron heat flux events, $J$. Geophys. Res., 92, 8519-8535, 1987.

Guio, P. and H. L. Pécseli, Phase space structures generated by an absorbing obstacle in a streaming plasma, Geophys. Res. Lett., 31, L03806, 2004.

Lin, R. P., D. L. Mitchell, D. W. Curtis, K. A. Anderson, C. W. Carlson, J. McFadden, M. H. Acuña, L. L. Hood, and A. Binder, Lunar surface magnetic fields and their interaction with the solar wind: Results from Lunar Prospector, Science, 281, 1480-1484, 1998.

McComas, D. J., J. T. Gosling, J. L. Phillips, S. J. Bame, J. G. Luhmann, and E. J. Smith, Electron heat flux dropouts in the solar wind: Evidence for interplanetary magnetic field reconnection?, J. Geophys. Res., 94, 6907-6916, 1989.
Mukai, T., S. Machida, Y. Saito, M. Hirahara, T. Terasawa, N. Kaya, T. Obara, M. Ejiri, and A. Nishida, The low energy particle (LEP) experiment onboard the GEOTAIL satellite, J. Geomag. Geoelectr., 46, 669-692, 1994.

Nakagawa, T., Y. Takahashi, and M. Iizima, GEOTAIL observation of upstream ULF waves associated with lunar wake, Earth Planets Space, 55, 569-580, 2003.

Ness, N. F. and K. H. Shatten, Detection of interplanetary magnetic field fluctuations stimulated by the lunar wake, J. Geophys. Res., 74, 64256438, 1969.

Ness, N. F., K. W. Behannon, H. E. Taylor, and Y. C. Whang, Perturbations of the interplanetary magnetic field by the lunar wake, J. Geophys. Res. 73, 3421-3440, 1968.

Ogilvie, K. W., J. T. Steinberg, R. T. Fitzenreiter, C. J. Owen, A. J. Lazarus, W. M. Farrell, and R. B. Torbert, Observation of the lunar plasma wake from the WIND spacecraft on December 27, 1994, Geophys. Res. Lett., 23, 1255-1258, 1996.

Owen, C. J., R. P. Lepping, K. W. Ogilvie, J. A. Slavin, W. M. Farrell, and J. B. Byrnes, The lunar wake at $6.8 \mathrm{R}_{L}$ : WIND magnetic field observations, Geophys. Res. Lett., 23, 1263-1266, 1996.

Phillips, J. L., J. T. Gosling, D. J. McComas, S. J. Bame, and S. P. Gary, and E. J. Smith, Anisotropic thermal electron distributions in the solar wind, J. Geophys. Res., 94, 6563-6579, 1989.

Pilipp, W. G., H. Miggenrieder, M. D. Montgomery, K.-H. Mühlhäuser, H. Rosenbauer, and R. Schwenn, Characteristicd of electron velocity distribution functionss in the solar wind derived from the Helios plasma experiment, J. Geophys. Res., 92, 1075-1092, 1987.

Schubert, G. and B. R. Lichtenstein, Observations of moon-plasma interactions by orbital and surface experiments, Rev. Geophys. Space Phys., 12, 592-626, 1974.

T. Nakagawa (e-mail: nakagawa@tohtech.ac.jp) and M. Iizima (e-mail: iizima@stpp1.geophys.tohoku.ac.jp) 